\title{
Pertussis im Neugeborenen- und Säuglingsalter - Kann ein Anstieg der Fallzahlen und Todesfälle verhindert werden?
}

\author{
Pertussis in Newborns and Infants - Can an Increase in Number of Cases and Deaths \\ be Prevented?
}

Wir berichten über den fatalen Verlauf einer Pertussis-Infektion eines ehemaligen Frühgeborenen.

\section{Anamnese}

Der Junge wurde mit einem Gestationsalter von 32 5/7 SSW per sekundärer Sectio aufgrund einer schweren Präeklampsie der Mutter, einer 36-jährigen Erstgravida, geboren. Eine unvollständige Lungenreifeinduktion mit Betamethason war durchgeführt worden. Nach Sectio in Intubationsnarkose zeigte das Kind eine verzögerte Adaptation, konnte jedoch mittels nicht-invasiver Beatmung stabilisiert werden. Die Geburtsmaße lagen im Normbereich. Im Alter von 20 Lebensstunden wurden bei Atemnotsyndrom eine Intubation und Surfactant-Gabe erforderlich. Bereits am Folgetag konnte das Kind extubiert werden und war ab dem sechsten Lebenstag ohne Atemunterstützung stabil. Nach einem insgesamt komplikationsarmen Verlauf konnte der Junge mit 36 1/7 SSW nach Hause entlassen werden.

Sechs Tage später erfolgte die stationäre Wiederaufnahme zur Rektumbiopsie bei Stuhlentleerungsstörung. Nebenbefundlich zeigte sich eine Atemwegsinfektion ohne Fieber. Die Mutter war aufgrund von Husten seit einigen Wochen bereits mehrfach in einer Notaufnahme vorstellig geworden; laborchemische Untersuchungen und eine Röntgen-Thorax-Aufnahme der Mutter waren unauffällig gewesen.

\section{Klinischer Befund}

Der Junge präsentierte sich initial mit einer Atemwegsinfektion und benötigte aufgrund einer Hypoxämie eine Sauerstoffvorlage. Apnoen oder Hustenfälle wurden nicht beobachtet und der durchgeführte RSV-Schnelltest war negativ, sodass der Verdacht auf eine RSV-negative Bronchiolitis bestand. Am fünften Tag nach Wieder- aufnahme kam es zu einer plötzlichen klinischen Verschlechterung mit Tachypnoe (95/min) und subkostalen Einziehungen, die eine Verlegung auf die Intensivstation (PICU) erforderlich machte.

\section{Diagnostik}

Bei PICU-Aufnahme zeigte sich die vorbestehende Leukozytose von $29 \mathrm{G} / \mathrm{I}$ progredient auf $59 \mathrm{G} / \mathrm{l}$ (37\% Lymphozyten) bei normwertigem CrP. Ein erneut durchgeführter RSV-Schnelltest war negativ; ausführliche weitere virologische und mikrobiologische Diagnostik inklusive MultiplexPCR und Pertussis-Serologie wurde in die Wege geleitet. In der initialen RöntgenThorax-Aufnahme zeigte sich das Bild einer zentralen Pneumonie.

\section{Therapie und Verlauf}

Auf der PICU wurde eine antibiotische Therapie mit Ampicillin (150 mg/kg/d in 3 ED) und Clarithromycin $(30 \mathrm{mg} / \mathrm{kg} / \mathrm{d}$ in $2 \mathrm{ED}$ ) begonnen. Die Leukozytose war progredient bis maximal $90 \mathrm{G} / \mathrm{l}$. Die Tachydyspnoe besserte sich unter CPAP-Therapie nicht, sodass noch am Tag der PICU-Aufnahme eine Intubation notwendig wurde. Unter HFO-Beatmung mit hohen Beatmungsparametern kam es zunächst zu einer Stabilisierung, im Verlauf des nächsten Tages jedoch zu einer zügigen globalen respiratorischen Verschlechterung, die eine weitere Intensivierung der Beatmung notwendig machte (MAD bis $24 \mathrm{cmH2O}$, Amplitude bis 60, Frequenz $9 \mathrm{~Hz}$, FiO2 bis 1,0). Bei Zeichen einer pulmonalen Hypertonie mit zunehmender Rechtsherzbelastung erhielt das Kind NO inhalativ und Prostaglandine intravenös. Zudem wurde 2-mal Surfactant verabreicht, was jeweils kurzzeitig zu einer verbesserten Oxygenierung führte. Bei weiterer respiratorischer Verschlechterung mit einem Oxygenierungsindex von 30 und zunehmender hämodynamischer Instabilität mit therapierefraktärer arterieller Hypotonie (Suprarenin
$0,6 \mu \mathrm{g} / \mathrm{kg} / \mathrm{min}$, Noradrenalin 0,6 $\mathrm{cg} / \mathrm{kg} / \mathrm{min}$, Vasopressin 0,02 IE/kg/h, HydrocortisonEinmalgabe, Transfusion eines Erythrozytenkonzentrats) erfolgte die notfallmäßige Verlegung in ein ECMO-Zentrum. Bei Ankunft wurde bei rasch progredienter Rechtsherzdekompensation unmittelbar eine VA-ECMO-Implantation durchgeführt. Oxygenierung und Ventilation waren problemlos möglich, jedoch gelang es aufgrund eines ausgeprägten Kapillarlecks nicht, eine stabile Kreislaufsituation zu etablieren. Das Kind verstarb am Folgetag an einem therapierefraktären Kreislaufversagen. Post mortem wurde der Befund der positiven PertussisPCR bei der Mutter - deren Symptome erst retrospektiv in vollem Umfang bekannt wurden - übermittelt. Auch im Trachealsekret des Kindes, im Labor eingegangen am Tag der Verlegung, war Pertussis-DNA nachweisbar.

\section{Diskussion}

Pertussis, eine durch das obligat humanpathogene Bakterium Bordetella pertussis ausgelöste Infektionskrankheit, ist weiterhin eine der am schlechtesten kontrollierten impfpräventablen Erkrankungen weltweit. Gründe sind unter anderem die schlechte Immunogenität des heute verwendeten azellulären Impfstoffes (aP), eine fehlende lebenslange Immunität auch nach Infektion oder Ganzkeim-Impfung, eine Inkubationszeit von bis zu drei Wochen, die hohe Kontagiosität sowie das Vorkommen von Trägertum und symptomarmen Infektionen. Der Impfschutz nach aP-Impfung nimmt ab dem vierten Jahr deutlich ab und ist sieben Jahre nach erfolgter Impfung kaum oder nicht mehr nachweisbar [Knuf M. Krankenhhyg up2date 2019; 14: 27-45]. 2014 bis 2018 gab es in Deutschland jährlich im Median 15,7 Fälle/100 000 Einwohner. Die Inzidenz ist stark altersabhängig und in der Gruppe der unter Einjährigen am höchsten (51,6 Fälle/100 000 Einwohner). 2016 wurden 
drei, 2014 bis 2018 zwei Todesfälle bei Säuglingen in Deutschland an das RKI gemeldet.

Bei Neugeborenen und Säuglingen verläuft die Infektion meist atypisch und häufig schwer. 18\% der Säuglinge mit Pertussis-Infektion müssen intensivmedizinisch behandelt werden. Klinisch führend sind die Komplikationen, Apnoen, die bei $61 \%$ aller hospitalisierten Säuglinge auftreten, Pneumonie (23\%) und Krampfanfälle (1\%) [DGPI, D. G. f. P. I., 2018; 7. vollständig überarbeitete Auflage: 880]. Vorherrschender Laborwert ist eine Leukozytose durch Lymphozytose. Die Mortalität bei intensivpflichtiger Pertussis-Infektion im Kindesalter beträgt 9,4\%; das relative Risiko für ein Versterben bei Leukozytose $>50$ G/l wird mit 9,8 angegeben [Namachivayam P et al., Pediatr Crit Care Med 2007; 8: 207211] [Berger JT et al., Pediatr Crit Care Med 2013; 14: 356-365]. Verursacht wird die Leukozytose durch das Pertussis-Toxin (PT), welches neben einer Inhibierung der Lymphozyten-Diapedese zu einer Inhibierung von GProteinen in Lunge und Herz führen kann. Unter Umständen ist dies die eigentliche Ursache für das respiratorische und kardiale Versagen, während die Leukozytose ein Marker für die PT-Aktivität ist [Winter K et al., Clin Infect Dis 2015; 61: 1099-1106]. Leukozytose sowie Hypoxämie durch Pneumonie und Apnoen begünstigen eine unter Umständen irreversible pulmonale Hypertonie (PHT), die bei der Mehrzahl der Pertussis-Todesfälle im Kindesalter auftritt.

Erkrankungsschwere und Progression der Erkrankung sind von transplazentar übertragenen mütterlichen oder Impfantikörpern des Säuglings, Bakterienlast, Koinfektionen, Gestationsalter sowie genetischen Faktoren des Pathogens und des Patienten abhängig. Bei Neugeborenen kann das erhöhte Vorkommen CD 71-positiver Zellen zu einer verringerten Phagozytose und Zytokinbildung führen. Aus Tierstudien ist zudem bekannt, dass ein Mangel an antimikrobiellen Peptiden mitverursachend für die besondere Schwere des Verlaufs einer Pertussis-Infektion bei Ferkeln sein kann. Dies könnte, auf den Menschen übertragen, eine Erklärung für die fulminant verlaufenden Pertussis-Septitiden im Neugeborenen- und Säuglingsalter sein. Prognostische Hinweise für einen schweren Verlauf und damit Indikatoren für eine PICU-Verlegung sind eine fulminante Pneumonie, Tachypnoe, Tachykardie, Leukozytose oder ein schneller Anstieg der Leukozyten [Murray EL et al., J Pediatric Infect Dis Soc 2013; 2: 1-6].

Bei jedem Verdacht auf eine Pertussis-Infektion im Neugeborenen- oder Säuglingsalter sollten frühzeitig Makrolide (bei Neugeborenen Azithromycin, bei Säuglingen Azithromycin, Erythromycin oder Clarithromycin) zum Einsatz kommen, da sie im späteren Stadium der Erkrankung keinen entscheidenden Einfluss mehr auf den Krankheitsverlauf haben. Eine Blutaustauschtransfusion kann bei jungen Säuglingen die Leukozytose sowie das zirkulierende PT reduzieren [1]. Die Mortalität einer ECMO-Therapie bei Pertussis-Infektion ist hoch (>70\%) und deutlich höher, als bei RSV-Infektion (20\%) [Domico M et al., Pediatr Crit Care Med 2018; 19: 254-261]. Vermutlich sind die verlängerte Passagezeit der Leukozyten im pulmonalen Gefäßbett und ihre hohe Anzahl Ursache der beobachteten Therapieresistenz auf die üblichen Therapieoptionen (NO, ECMO) einer PHT.

Eine Pertussis-Impfung in der Schwangerschaft wird seit 2012 in den USA und in Großbritannien empfohlen. Mechanismen sind der passive Antikörper-Transfer über Plazenta und Muttermilch sowie die reduzierte Exposition der Mütter. Die Impfung gilt als sicher und hat eine hohe Wirksamkeit, einerseits, Pertussis-Infektionen zu verhindern und andererseits, bei eingetretener Infektion, deren Verlauf abzuschwächen. Die Impfung in graviditate gilt derzeit als effektivste Maßnahme zur Verhinderung schwerer Pertussis-Infektionen im Säuglingsalter [Gkentzi D et al., Arch Dis Child Fetal Neonatal Ed 2017; 102: F456-F463]. In den USA wird sie zwischen der 27. und 36., in Großbritannien zwischen der 16. und 32. SSW empfohlen; bester Impfzeitpunkt scheint die 30. SSW zu sein. [Healy CM et al., JAMA 2018; 320: 1464-1470]. Während die Entscheidung der STIKO aussteht, befürwortet die Kommission für Infektionskrankheiten und Impffragen der Deutschen Akademie für Kinder- und Jugendmedizin e.V. die Einführung einer Empfehlung zur Pertussis-Impfung für schwangere Frauen ab dem zweiten Trimenon. Die Mutter des an einer Pertussis-Sep- sis verstorbenen ehemaligen Frühgeborenen aus dem Fallbericht war nicht gegen Pertussis geimpft.

Die zeitgerechte Impfung aller Säuglinge sowie die Überprüfung des Impfstatus von Familienmitgliedern und engen Kontaktpersonen aller Neugeborenen sind weitere unerlässliche Maßnahmen zur Vermeidung schwerer Pertussis-Infektionen im Neugeborenen- und Säuglingsalter. Eine teilweise Impfpflicht kann die Impfbereitschaft für weitere, freiwillige Impfungen reduzieren [Betsch C et al., Eur ] Public Health 2016; 26: 378-381]. Demnach könnte die Einführung einer isolierten Masern-Impfpflicht die Impfbereitschaft gegen Pertussis senken und so die bereits jetzt schon niedrige Herdenimmunität gegen Pertussis weiter verringern.

Fazit Bei einer fulminant verlaufenden respiratorischen Erkrankung eines Neugeborenen oder Säuglings ist die schwere Infektion mit Bordetella pertussis eine wichtige Differenzialdiagnose. Eine sofortige Makrolidtherapie und eventuell eine Austauschtransfusion bieten die einzigen therapeutischen Optionen. Die Impfung, auch in der Schwangerschaft, bietet eine effektive Prophylaxe. Die Impfung in der Schwangerschaft stellt derzeit die effektivste Maßnahme zur Verhinderung schwerer Pertussis-Infektionen im Neugeborenen- und Säuglingsalter dar. Die Einführung einer Impfempfehlung könnte somit einen Anstieg der Fallzahlen und Todesfälle verhindern.

\section{Stellungsnahme zur}

\section{Autorenschaft}

Eine Überarbeitung des Manuskripts erfolgte durch A. Flemmer, M. Klemme, A. Jakob, S. Ulrich und A. Hohnecker. Durch S. HerberJonat wurde ich auf die Thematik „Pertussis im Neugeborenen- und Säuglingsalter“ und den Fall des ehemaligen Frühgeborenen aufmerksam gemacht. Sie überarbeitete zudem Inhalte, die sich im Diskussionsteil finden. A. Hohnecker stellte wichtige Informationen zum klinischen Verlauf des Kindes vor Verlegung in das ECMO-Zentrum zur Verfügung. 


\section{Interessenkonflikt}

S. Ulrich erhielt eine Forschungsförderung von Novartis Pharma GmbH, von Astellas Pharma GmbH, von dem Verein zur Förderung von Wissenschaft und Forschung (medizinische Fakultät der Ludwig-Maximilians-Universität München) und von der Gerd-Killian Projektförderung (DGPK, „Deutsche Herzstiftung“). Zusätzlich erhielt sie Honorar für Vorträge von Novartis Pharma GmbH. Die anderen Autoren geben an, dass kein Interessenkonflikt besteht.

\section{Literatur}

[1] Winter K, Zipprich J, Harriman K et al. Risk Factors Associated With Infant Deaths From Pertussis: A Case-Control Study. Clin Infect Dis 2015; 61: 1099-1106

\section{Autoren}

Katrina Harnacke ${ }^{1}$, Mathias Klemme ${ }^{1}$, Susanne Herber-Jonat ${ }^{1}$, Andre Jakob ${ }^{2}$, Sarah Ulrich ${ }^{2}$, Alexander Hohnecker ${ }^{3}$, Andreas Flemmer ${ }^{1}$

\section{Institute}

1 Neonatologie des Dr. von Haunerschen Kinderspitals am Perinatalzentrum Großhadern, Klinikum der LMU München

2 Abteilung Kinderkardiologie und Pädiatrische Intensivmedizin, Klinikum der LMU München

3 Kinderklinik des Klinikums Dritter Orden, München

\section{Korrespondenzadresse}

Dr. med. Katrina Harnacke

Dr. von Haunersches Kinderspital

Klinikum der LMU München

Lindwurmstraße 4

81377 München

Tel.: + 49/894/40052 811

Fax: + 49/894/40057 749

katrina.harnacke@med.uni-muenchen.de

\section{Bibliografie}

DOI https://doi.org/10.1055/a-1007-8768

Online-Publikation: 30.9.2019

Klin Padiatr 2020; 232: 37-39

(c) Georg Thieme Verlag KG Stuttgart · New York ISSN 0300-8630 